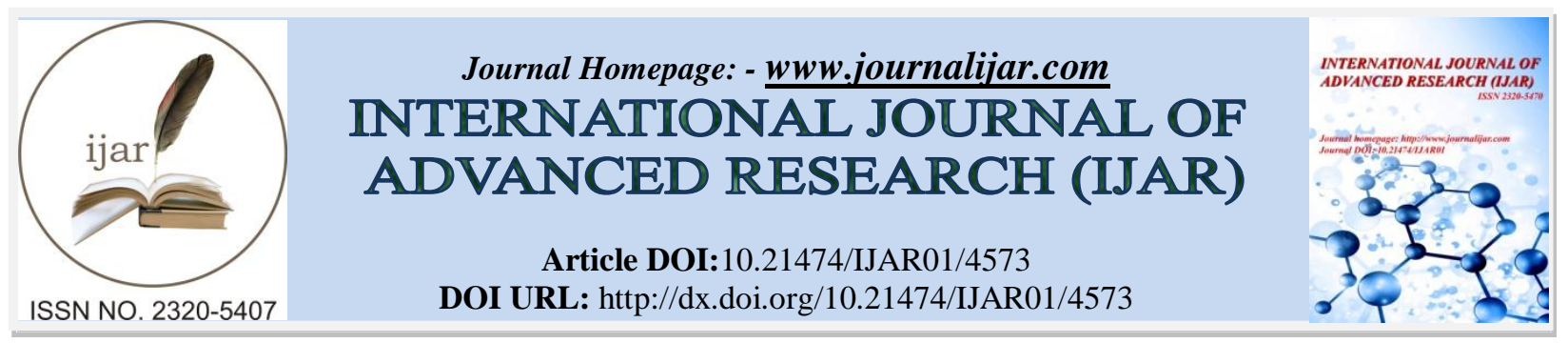

RESEARCH ARTICLE

\title{
A HYBRID SELF-ORGANIZING MAP WITH FUZZY K-MEANS ALGORITHM FOR BRAINTUMOR IDENTIFICATION AND ANALYSIS USING MAGNETIC RESONANCE BRAIN IMAGES.
}

Isselmou Abd El Kader, Shuai Zhang and Guizhi Xu.

Department of Biomedical Engineering, Hebei University of Technology, Tianjin, 300130, China.

\section{Manuscript Info}

\section{Manuscript History}

Received: 22 April 2017

Final Accepted: 24 May 2017

Published: June 2017

Key words:-

MRI, SOM-FKM, Tumor Identification,

Segmentation, Accuracy Detection,

Jaccard Index, Dice Overlap Index,

Sensitivity, Specificity, recall.

\section{Abstract}

Medical image processing is considered the area a great challenge for researchers because of the complexity of this area and its importance to help doctors to diagnose patients effectively. Here we will base on magnetic resonance brain images processing trough the image processing technique is hybrid self-organizing map (SOM) with fuzzy k-means (FKM) algorithm, which gives successfully on tumor and excellent areas within the brain tissue, we propose improved algorithm is accurate and efficient in term of accuracy detection, n Jaccard coif, nDice (DOI), sensitivity, specificity, recall, precision, performing segment. At the same time, the improved algorithm proposed provides the best information and functions, as well as the efficiency and accuracy to handle high input brain MR images.

Copy Right, IJAR, 2017,. All rights reserved.

\section{Introduction:-}

The tumor word cancer, also known as the tumor, refers to more than they need or should not have died later cause abnormal swelling of tissue during cell division [1]. Brain tumors can be organized by uncontrolled cell growth and reproduction of abnormal masses, through the mechanism of control normal cells appears selected. Two large individual identification in addition to their own style and vicious (benign and malignant) growth depends on the origin of the tumor (primary, metastatic).

Magnetic resonance imaging (MRI) to give the human body, especially the brain complicated structure, it depends on the results of analysis of the three-dimensional image of the NMR structure of the principles of the internal components within the heart and in the brain tissue. Traditional analysis of magnetic resonance images of medical examination is a very complex problem that requires a long time, so experts affect the accuracy of diagnosis and manual process is also an error -prone and inaccurate in terms of noise, therefore, the solution is also recommended image segmentation, which gives excellent results.

NandhaGopa [2] this automatic detection of brain tumors, can be divided in MRI (magnetic resonance), 95\% of the need to further improve the accuracy of the generated image of the brain. Vishnuvarthanan and Rajasekeran [3] recommend using basic fuzzy inference system automation and extracted tumor tissue segmentation. It deficiencies in computation time, PSNR, and MSE area, Guerrout et al [4] with medical image segmentation field theory of Markov, The algorithm for medical image segmentation process is only complete contrast enhancement in T1 and T1-weieghted images. $\mathrm{Li}$ and Chi used SOM, which requires the RMS value of reducing brain MR image segmentation. 
In this paper, we propose improved hybrid SOM-FKM algorithm for tumor identification in magnetic resonance images (MRI) we improved the previous results in terms accuracy detection, Jaccard Index, Dice Overlap Index (DOI), Sensitivity, Specificity,

\section{Materials:-}

In this work, we used 4 Magnetic resonance brain images FLAIR1, FLAIR2, FLAIR3, and FLAIR4 obtained using Siemens-Areas 1,5T from Florida University brain repository have been used to validate the proficiency of the proposed improved algorithm, the main property is inhibited by the proposed SOM-FKM algorithm.

\section{Methodology:-}

We proposed here improved hybrid SOM-FKM algorithm improve results, SOM used to assemble and reduced, the process begins with the introduction of magnetic resonance image of the brain (Input MR brain image) the primary treatment for the image using SOM (applying color Quantization, Training SOM, Classify Segmentation, Morphology cleaning, Tumor identification). The second step of image processing using improved hybrid SOMFKM applying (Segmentation with FKM, Tumor masking, Morphology tumor), after these two steps we get the area of the tumor using the SOM alone and improved hybrid SOM-FKM then compare the two results after analyze the results and get the performance of the SOM alone and improved hybrid SOM-FKM, shown in the figure.1.

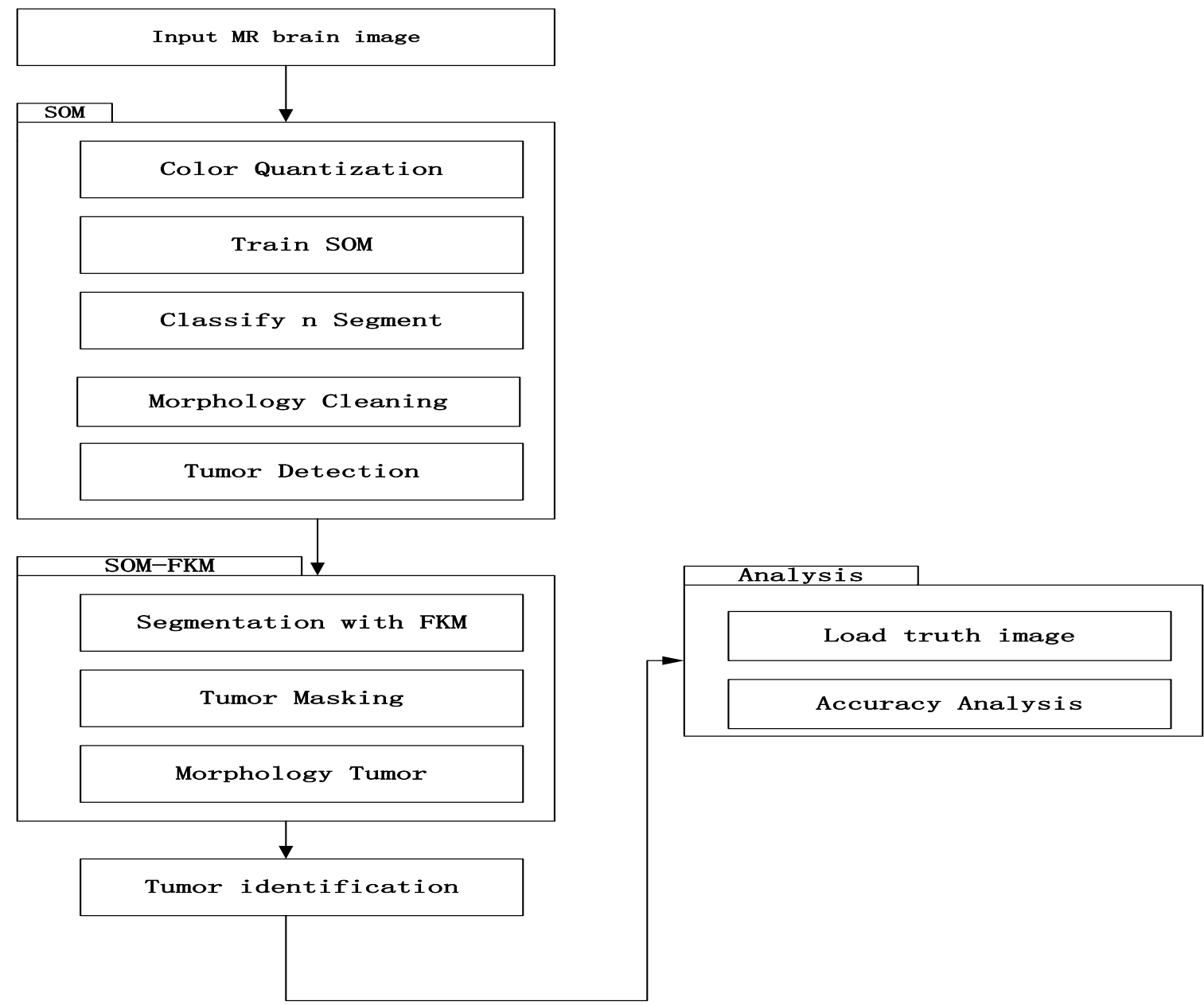

Figure.1:- Architecture of Hybrid SOM-FKM algorithm

3.1Self-organizing map algorithm (SOM):-

Probability density function SOM processing algorithm voxel intensity to initiate and help process model and divided by SOM at the start began to be realized and contribute to the analytic peak, which can be greatly found in segmentation process [5, 6, and 7]. The SOM algorithm is explained as follows: 
Let $X \in R^{d}$, were ${ }^{d}$ represents the diversified input data and $\mathrm{X}$ is the input vector, the warning unit is calculated in each iterative steps as,

$$
\bigcup_{\omega}(t)=\arg \min _{i}\left\{\left\|x(t)-\omega_{i}(t)\right\|\right\}
$$

$x \in X$, Where $\mathrm{x}(\mathrm{t})$ is defined as the input vector at time instant and $\omega_{i}(t)$ are the sample vectors of the relevant units. The unit is very close the input vector $\bigcup_{\omega}(t)$ is represented by the winner until the prototype or sample and associated vectors are sequentially updated $[5,6]$. Near the until won the SOM adaptive learning process by updating the prototype until the end, this process is described as:

$$
\omega_{i}(t+1)=\omega_{i}(t)+\alpha(t) h_{\cup_{i}}(t)\left(x(t)-\omega_{i}(t)\right)
$$

Here $\alpha(t)$ is the exponential decay learning factor and $h_{\cup_{i}}(t)$ is the neighborhood function related to the unit't'. As near unit contains a small number of units features two factors and learns to live with respect to reducing the time and adaptability slower prototype [7].

$$
h_{\cup_{i}}(t)=e^{-\left(\left\|\gamma_{u-\gamma_{i}}\right\|^{2 / 2 \gamma(t)^{2)}}\right.}
$$

FKM utilizes the output from SOM performs the clustering operations as specified in [22]. Prototype $\omega_{i}$ is the input fed to FKM algorithm and the following process takes place sequentially;

$$
J_{k}=\sum_{j=1}^{k} \sum_{i=1}^{N} u_{i}^{m} d_{i j}
$$

Here, $\mathrm{k}$ describes the total number of iterations, $U_{i j}$ represents the membership function and it's calculation is described in Eq (5), $d_{i j}$

$$
U_{i j}=\left[\left(d_{i j}\right)^{1 /(m-1)} \sum_{1=1}^{k}\left(\frac{1}{d_{i j}}\right)^{1 /(m-1)}\right]^{-1}
$$

In Eq (6), $C_{j}$ denotes the cluster center, $C_{j}$ can also be called cluster representative.

$$
C_{j}=\frac{\sum_{N}^{i=1} U_{i j}^{m} x i}{\sum_{N}^{i=1} U_{i j}^{m}}
$$

Eq (7) explicates the sum function of membership values of each data point and it should be equal to one.

$$
\sum_{j=1}^{k} U_{i j}=1 \text {, For } f=1 \text { to } \mathrm{N}
$$

Function $J_{K}$, FKM algorithm explained obtained from the final segmentation results. As a whole, SOM dramatically by providing a level of segmentation algorithms supported FKM.

\subsection{Neural Networks:-}

Nerve organs networks are formulated using functionality exhibited by the biological nervous systems, a significant place in the combined mode where these neurons, plus they were later distributed to input information to perform the study. Neuron control internal working procedures finally opened an optimized result. Some neural network method for processing medical image analysis, especially with regard to the image segmentation

\subsection{Morphological operations:-}

Here the morphological operations such as binary dilation, binary erosion are applied to the image. Binary closing and binary opening operations are applied to image using the Equations 8 and 9

$$
\begin{gathered}
A \circ B=(A \Theta B) \oplus B \\
A \bullet B=(A \oplus B) \Theta B(9)
\end{gathered}
$$




\subsection{Fuzzy K-means (FKM) Algorithm:-}

Fuzzy K-means algorithm, also known as FKM, the company's behavior competitors, FCM formula, as mentioned ALTER, el [8], fluorine rubber partition for given input image is divided into several ' $\mathrm{k}$ ' cluster (divided image voxel).

$$
J=\sum_{J=1}^{K} \sum_{i=1}^{N} u_{i, j}^{m} d_{i j}
$$

Here, $N$ represents a number of data points or voxels within the type MR brain image. T refers to the quantity of groupings form by FKM. $i, j$ Make clear the series and columns of the input image. $i, j$ Defines the account functions of FKM with fuzziness coefficient $\mathrm{m}$ (fuzziness coefficient is extracted from the overlapping of clusters). $d_{i j}$ is the squared Euclidean distance between the pixel $x_{i}$ and cluster representative $C_{j}$ (cluster center or centroid value). FKM algorithm converges faster and requirements for processing an image less computing time. It has handed over FKM limited range of tissues handled recommended method is to use the data and overcome SOM.

\subsection{Parameters:-}

\subsubsection{Accuracy Detection:-}

Accuracy, reliability also called segmentation; it is used to determine the effectiveness of the segmentation algorithm evaluation variables. The accuracy is denoted in Eq (11).

$$
\text { Accuracy }=\left(\frac{k}{m \times n}\right) \times 100
$$

Here, $k$ ' is the total number of pixels present in the segmented output image, ' $m$ ' and $n$ are the rows and columns presents in the input image. Persistently the total number of pixels present in the input image.

\subsubsection{Jaccard (Tanimoto) Index:-}

Jaccard Index it is present in the input image and the intersection (A) of the voxel value and set the rate and the resulting segmented image $(\mathrm{B})$ between functions.

$$
J(A, B)=\frac{S(A \cap B)}{S(A \cup B)}
$$

\subsubsection{Dice Overlap Index (DOI):-}

DOI value is expressed with Jaccard index J(A, B). DOI defines the overlapping function of the type image (A) and segmented output image (B) [9].

$$
D(A, B)=2 \times \frac{J(A, B)}{1+J(A, B)}
$$

\subsubsection{Sensitivity:-}

Sensitivity value refers to the input image appropriate division or classification [10] defined error rate success in accurately identify tumors areas. This is explained as:

\subsubsection{Specificity:-}

$$
O F=\frac{T P}{T P+F N}
$$

Specificity defines specific word or algorithms to classify segments of normal tissue are present in a region in the input image capability. Specificity is believed using Eq (15).

$$
\text { Specificity }(\sigma)=\frac{T N}{T N+F P}
$$

Here, $T N$ is the true negative value. It briefs the effective segmentation of non-tumor region or normal brain tissues by the algorithm.

\section{Experiment Results:-}

The operation was conducted experiments on four images from magnetic resonance imaging of the brain FLAIR1, FLAIR2, FLAIR3, FLAIR4 (A, B, C, D MR Brain Images), we will divide the results into two parts as follows: 
4.1 Results Self-Organizing Map (SOM) and Performance Evaluation:-

4.1.1 Results Self-Organizing Map (SOM):-

Table.1:- Segmentation Results from Self-Organizing Map (SOM)

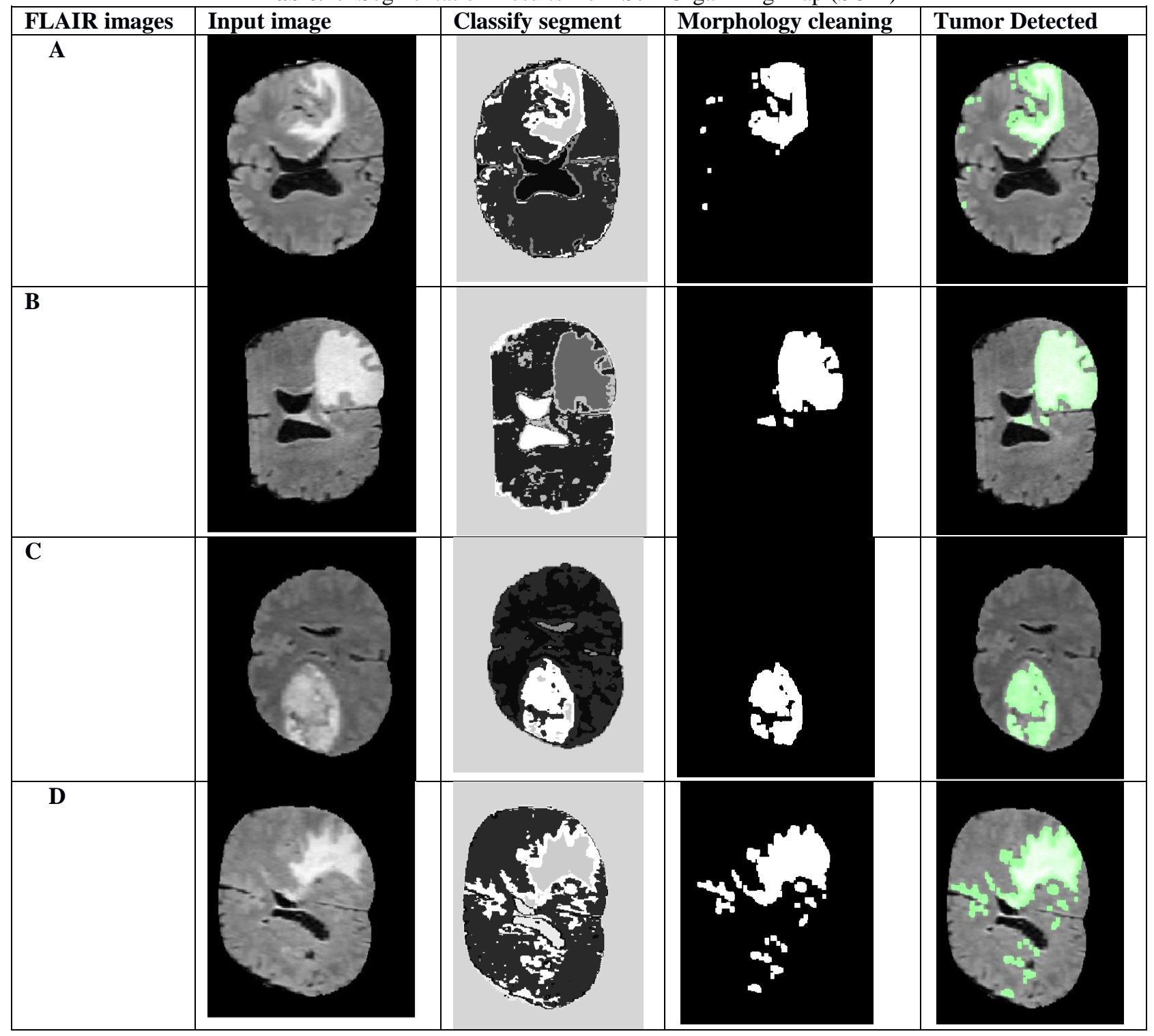

4.1.2Evaluation Performance of Self-Organizing Map (SOM):-

Table.2:- "Performance Evaluation for Self-Organizing Map (SOM)"

\begin{tabular}{|l|l|l|l|l|l|l|l|}
\hline $\begin{array}{l}\text { FLAIR } \\
\text { images }\end{array}$ & $\begin{array}{l}\text { Accuracy } \\
\text { detection \% }\end{array}$ & $\begin{array}{l}\text { Jaccard } \\
\text { Index }\end{array}$ & DOI & Sensitivity & Specificity & Recall & Precision \\
\hline A & 95,201 & 0,2843 & 0,5587 & 0,6939 & 0,6939 & 0,693 & 0,9790 \\
\hline B & 95,201 & 0,5076 & 0,3408 & 0,8128 & 0,8128 & 0,812 & 0,6027 \\
\hline C & 95,201 & 0,7580 & 0,8659 & 0,9871 & 0,9871 & 0,987 & 0,8839 \\
\hline D & 95,201 & 0,3001 & 0,6801 & 0,7743 & 0,7743 & 0,7743 & 0,9070 \\
\hline
\end{tabular}

4.2 Results Hybrid Self-Organizing Map with Fuzzy k-means Algorithm (SOM-FKM) and Performance Evaluation of SOM-FKM 


\subsubsection{Results Hybrid Self-Organizing Map with Fuzzy K-means Algorithm (SOM-FKM).}

Table.3:- " Segmentation Results from Hybrid SOM-FKM Algorithm"

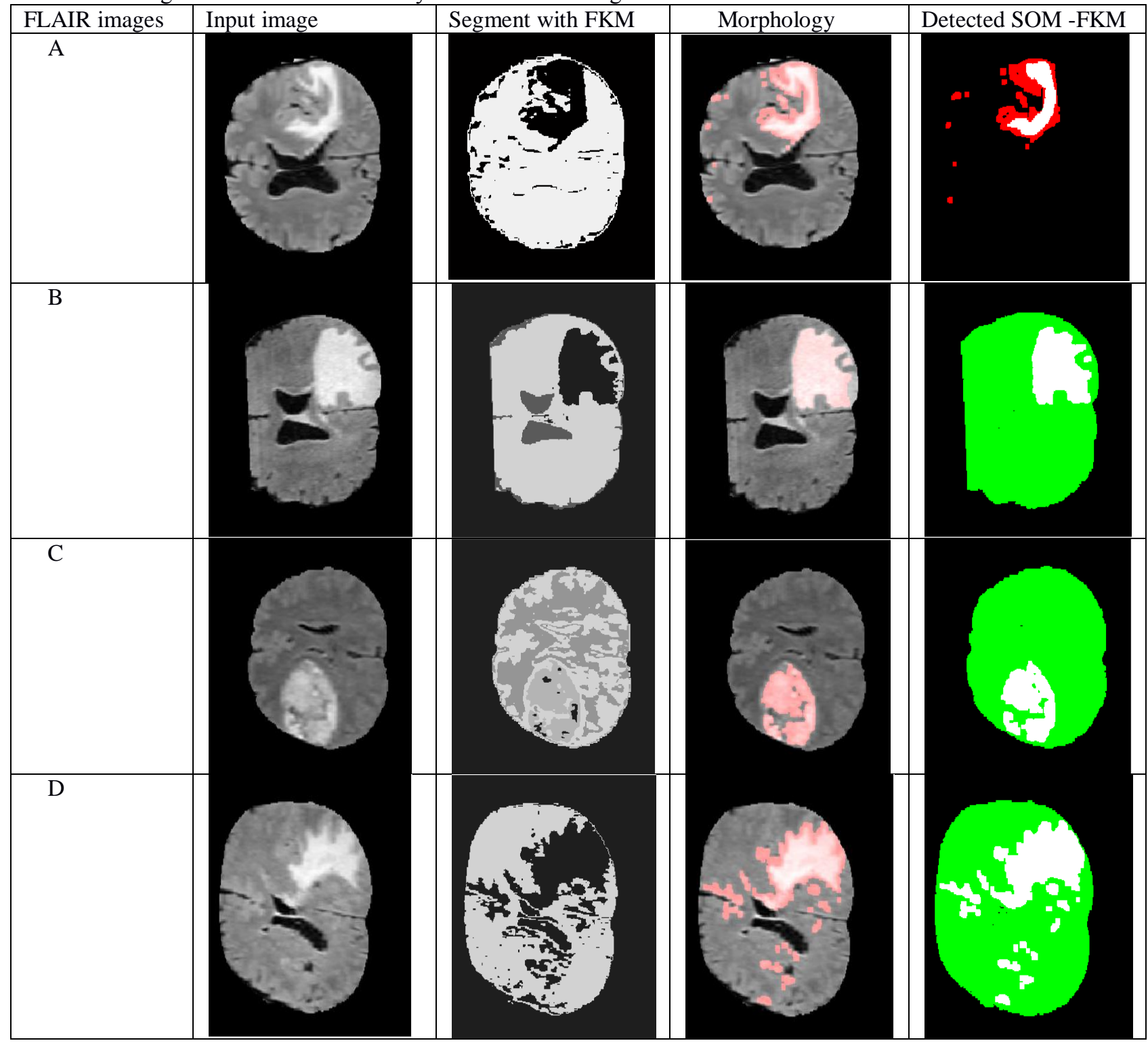

\subsubsection{Performance Evaluation for SOM-FKM Algorithm:-}

Table.4:- "Performance Evaluation of Hybrid SOM-FKM Algorithm"

\begin{tabular}{|c|c|c|c|c|c|c|c|c|}
\hline $\begin{array}{c}\text { FLAIR } \\
\text { images }\end{array}$ & $\begin{array}{c}\text { Accuracy } \\
\text { Detection \% }\end{array}$ & $\begin{array}{c}\text { Jaccard } \\
\text { Index }\end{array}$ & DOI & Sensitivity & Specificity & Recall & Precision & $\begin{array}{c}\text { Performing } \\
\text { Segment }\end{array}$ \\
\hline A & 99,940 & 0,7862 & 0,2830 & 0,9837 & 0,9837 & 0,6937 & 0,9789 & $-1,905$ \\
\hline B & 99,940 & 0,5076 & 0,8678 & 0,9877 & 0,9877 & 0,8877 & 0,9811 & 2,007 \\
\hline C & 99,940 & 0,7580 & 0,8693 & 0,9871 & 0,9871 & 0,9871 & 0,8839 & 0,7923 \\
\hline D & 99,940 & 0,3002 & 0,6801 & 0,9843 & 0,9843 & 0,7743 & 0,9070 & $-1,101$ \\
\hline
\end{tabular}

\section{Conclusion:-}

In this paper, we have improved hybrid algorithm SOM-FKM and given excellent results from the (accuracy detection, Jaccard Index, DOI, Sensitivity, Specificity, Recall, and Precision). Show the effectiveness of the 
improved algorithm in Table.3 (images A, B, C, D) and Table.4 were the performance of the algorithm S0M-FKM, as well as when compared to the results SOM alone or results in former researchers Nandha Gopa or Vishnuvarthanan and Rajasekerman. The purpose of the paper to help the doctors diagnose a patient in a short period of time and what is achieved with improved hybrid SOM-FKM.

\section{Acknowledgement:-}

This work was supported in the part by the natural science foundation of Hebei Province under Grant No: E2015202292 and No: E2015202050, high-level talent support project in Hebei Province under Grant No: C2015005012, key research, and development program under Grant No: 15272002 and No: 15275704.

\section{References:-}

1. Nandha Gopal, Automatic detection of brain tumor trough MR image, Int, J.Adv.Res. Comput. Commun. Eng,2 ( April (4)) (2013).

2. E, Ben George, M. Karman, MR brain image segmentation using bacterial foraging optimization algorithm, Int. J. Eng. Technol. 4 (Octobre-November (5)) (2012).

3. S. Aljahdali, E.A. Zanaty, Automatic fuzzy algorithms for reliable image segmentation, IJCA 19 (September (3)) (2012).

4. S. Aljahdali, E, A. Zanaty. Improving fuzzy algorithms for reliability image segmentation, IEEE (2010), 978-161284-732-0\11.

5. A. Ortiz, J.M. Gorriz, J. Ramirezb, D Salas-Gonzales, J.M. Llamas-Elvira, Two modified FCM framework for Improved brain MR image segmentation using SOM- based strategies, Appl. Soft Comput. 13 (2013) 26682682).

6. S.T. Welstead. Fractal and Wavelet Image Compression Techniques, SPIE Publication. 1999, pp. 155156,ISBN 978-0-8194-3503-3.

7. N. Thomos, N.V.Boulgouris, M.G Strintzis, Optimized transmission of JPEG2000 streamos over wireless channels, IEEE Trans. Images Process. 15 (1) (2006).

8. J. Yu, M.S. Yang, A generalized fuzzy clustering regularization model with optimality tests and model With optimality tests and model complexity analysis, IEEE Trans. Fuzzy Syst. 15 (5) (2007) 904-915.

9. S.Shen, W. Sandham, M. Granger, A.Sterr. MRI Fuzzy segmentation of brain tissue using neighborhood Attraction with neural network optimization. IEEE Trans. Inf. Technol. Biomed. 9(2005) 459-467.

10. A. Raid, A. Atwan, H. El-Bakry, R. Mostafa, H. Elminir, N. Mastorakis, A new approach for segmentation Of MR brain image, in; Proceedings of the WSEAS International Conference Environment, Medicine and Health Sciences, 2010 